\title{
The Morphological Evaluation of Walker 256 Tumors after Antiangiogenetic Therapy in Rats
}

\author{
Alexandra IRIMIE ${ }^{1 *}$, Attila Tamas SZORA ${ }^{2}$, Mihai SOCACIU ${ }^{2}$, Alexandru Flaviu TĂBĂRAN ${ }^{1}$, Raluca VIDRI- \\ GHINESCU ${ }^{1}$, Cornel CĂTOI ${ }^{1}$ \\ ${ }^{1}$ Department of Pathology, University of Agricultural Sciences and Veterinary Medicine, Cluj Napoca, \\ Romania \\ ${ }^{2}$ Ultrasonography Department, 3rd Medical Clinic,“Iuliu Haţieganu” University of Medicine and Phar- \\ macy, Cluj Napoca, Romania \\ *Corresponding author, e-mail: alexandra.irimie2010@gmail.com
}

Bulletin UASVM Veterinary Medicine 71(2) / 2014,

Print ISSN 1843-5270; Electronic ISSN 1843-5378

DOI:10.15835/buasvmcn-vm: 10558

\begin{abstract}
Sunitinib Malate is a multi-targeted receptor tyrosine kinase inhibitor, including the vascular endothelial growth factor receptors, and is used for treatment of pancreatic neuroendocrine, renal cell carcinoma and gastrointestinal stromal tumors. The formation of new blood vessels (angiogenesis) may consequently lead to the development of metastases and so the aim of this study was to evaluate the microvessel density (MVD) after sunitinib malate treatment. In this study 11 albino Wistar rats were inoculated subcutaneously with Walker 256 tumor (breast carcinoma cells) and after 5 weeks, 5 rats were administered sunitinib malate at a dosage of $40 \mathrm{mg} / \mathrm{kg}$ every 3 days for 2 weeks. After another 4 weeks the tumors were removed and morphologically evaluated (weighted, measured). The MVD determined and quantified by calculating the average between 3 random fields. In the tumoral stroma we calculated also the vascular area. The immunohistochemical analysis of the neovascularization was performed using an anti-rat antibody against CD34. The difference between tumor weights were statistical significant $(\mathrm{p}<0.05)(3.746 \pm 1.633 ; 1.019 \pm 0.812)$ but the tumor sizes and most importantly MVD between the control group and the experimental group were not statistical significant $(p>0.05)(32.55 \pm 18.83 ; 18.6 \pm 4.734)$. As a conclusion, the rats treated with Sunitinib malate did not show a lower microvessel density comparative to the untreated ones. The number of rats used in this research was too small and further studies are needed.
\end{abstract}

Keywords: Angiogenesis, MVD, Sunitinib Malate, Walker 256

\section{INTRODUCTION}

Angiogenesis defines the formation of new blood vessels. All tissues require a continuous supply of oxygen including the tumoral tissue. Oxygen is conducted to these tissues by blood capillaries: more capillaries can increase tissue oxygenation and thus enhance energy production; fewer capillaries can lead to hypoxia and even anoxia in the tissues (Adair and Montani, 2010). Also the formation of new blood vessels may lead to the development of metastases with the in-going of the malignant cells into the circulation. Thus the antiangiogenetic therapies are designed to reduce and control the growth of these new blood vessels and to induce the death of the tumor cells. The antiangiogenetic therapies are a possible cancer treatment.

Sunitinib Malate is a multi-targeted receptor tyrosine kinase inhibitor, including the vascular endothelial growth factor receptors, and is used for treatment of pancreatic neuroendocrine, renal cell carcinoma and gastrointestinal stromal tumors (FDA Approval summary).

For over 10 years, microvessel density (MVD) was used as a surrogate marker of tumoral angiogenesis and is the most commonly used techni- 
que to quantify intratumoral angiogenesis in breast cancer. It was first developed by Weidner et al. in 1991 and uses panendothelial immunohistochemical staining of blood microvessels (Factor VIII antigen, CD31, PECAM-1, or CD34, etc.) (Uzzan et al., 2004).

The aim of this study was to evaluate the microvessel density (MVD) after sunitinib malate treatment.

\section{MATERIALS AND METHODS}

In this study 11 albino Wistar rats were inoculated subcutaneously with Walker 256 tumor (breast carcinoma cells) and after 5 weeks, 5 rats were administered sunitinib malate at a dosage of $40 \mathrm{mg} / \mathrm{kg}$ every 3 days for 2 weeks. After another 4 weeks the tumors were removed, photographed and morphologically evaluated (weighted, measured).

\section{Histology}

The samples were fixed immediately after excision in $10 \%$ neutral buffered formalin for 24 hours and embedded in paraffin wax. Serial sections were cut at $4 \mu \mathrm{m}$ from each block and stained with hematoxylin and eosin (H\&E).

\section{Immunohistochemistry}

The immunohistochemical study was realised by using anti-CD34 antibodies (diluatia 1:10, R\&D Systems, Minneapolis, USA). Serial sections were cut at $4 \mu \mathrm{m}$ from each block and mounted on poly-L lysine coated slides. The immunohistochemical protocol was performed using the Leica Bond Max automatic system.

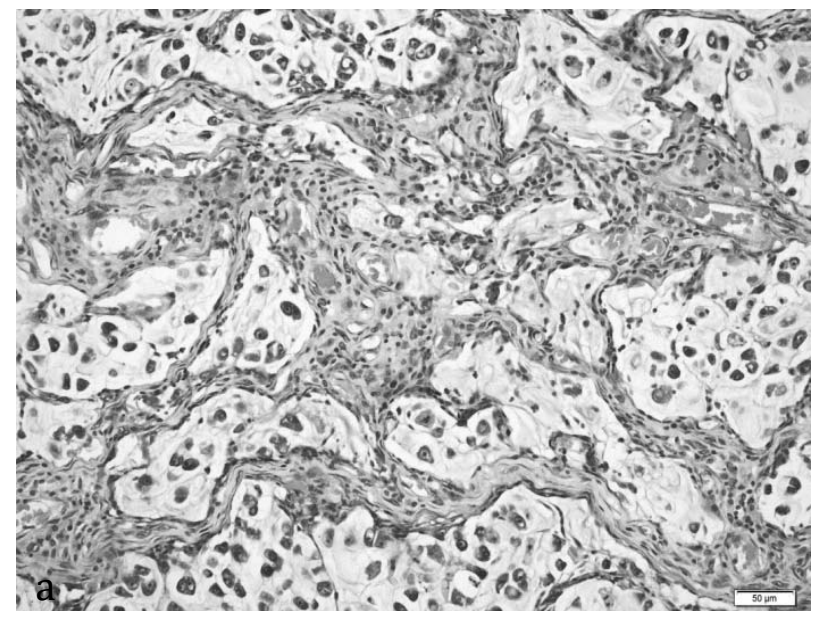

\section{Microvessel Density (MVD)}

The microvessel density was determined using light microscopy. The microscope used in this study was an Olympus BX41 microscope with an Olympus UC30 photo camera and an Olympus Stream Basic software. Using Weidner's approach (1991), 3 random fields were established by using a 10X objective lens (100X magnification), where the most capillaries ("hot spot") can be observed.

Tab. 1. Tumor weights

\begin{tabular}{ll}
\hline $\begin{array}{l}\text { Weight }(\mathrm{g}) \text { - untreated } \\
\text { group }\end{array}$ & $\begin{array}{l}\text { Weight }(\mathrm{g}) \text { - Experimental } \\
\text { Group }\end{array}$ \\
\hline 3.577 & 0.290 \\
\hline 3.490 & 1.546 \\
\hline 4.020 & 1.148 \\
\hline 6.287 & $0.357+1.649$ \\
\hline 3.936 & 0.103 \\
\hline & 1.762 \\
\hline
\end{tabular}

The capillaries in both the tumor stroma and the tumor capsule were counted using a 20X objective lens (200X magnification). After the capillaries were counted, the average was calculated between these fields. At the same time the area of the large and medium large vessels was calculated in percentage (vascular area reported to the total area of the field). The total area of the field was $377956.98 \mu \mathrm{m}^{2}$.

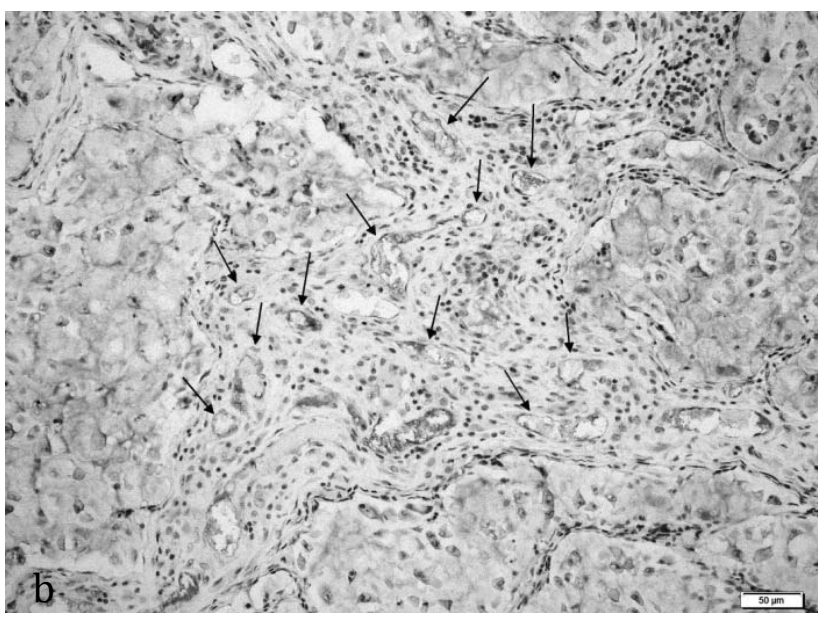

Fig. 1. The Untreated Group (a) H\&E stain. (b) Immunohistochemical staining with antibody against CD34. Positive capillary endothelial cells indicated by black arrows. Note the cytoplasmatic membranes of the tumor cells also marked. Magnification at 200X. 

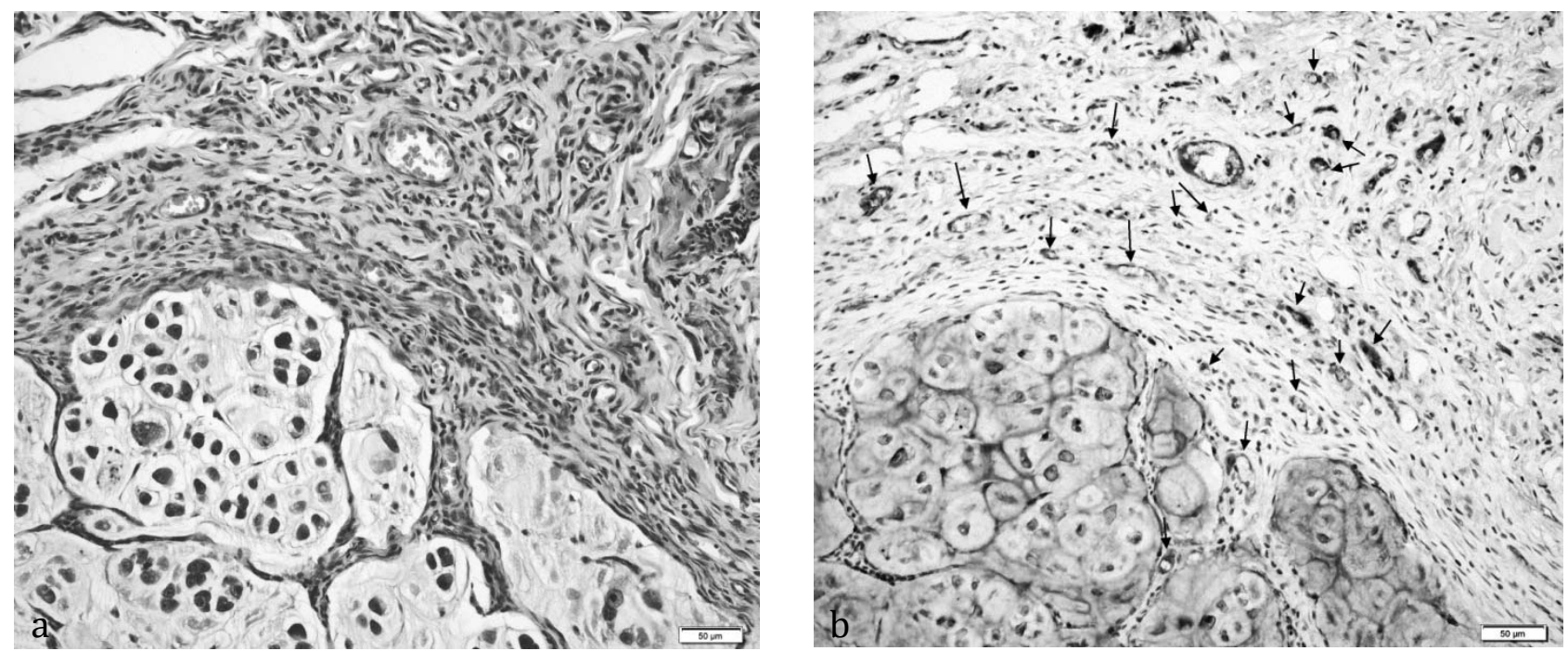

Fig. 2. The Experimental Group. (a) H\&E stain (b) Immunohistochemical staining with antibody against CD34. Positive capillary endothelial cells indicated by black arrows.

Tab. 2. The microvessel density (MVD) and vascular area in the untreated group

\begin{tabular}{cc}
\hline MVD & Vascular Area (\%) \\
\hline 32.33 (S 16+ C 81) & 3.44 \\
32 (S 25 + C 71) & 3.17 \\
19 (S 7 + C 50) & 1.04 \\
24.33 (S 37 + C 36) & 2.33 \\
18.66 (S 37 +C 19) & 6.35 \\
69 (S 70 + C 137) & 4.56 \\
\hline
\end{tabular}

S - stroma ; C - capsule

Statistics: Independent group t-tests were used to compare two groups in regard to the categorical data, by using Microsoft Excel.

\section{RESULTS AND DISCUSSIONS}

After the tumors were weighted there could be seen a significant difference between the experimental group and the untreated group (table 1).

The difference between tumor weights were statistical significant $(\mathrm{p}<0.05) \quad(3.746 \pm \mathbf{1 . 6 3 3}$; $\mathbf{1 . 0 1 9} \pm \mathbf{0 . 8 1 2}$ ), but the tumor sizes and volumes were not statistical significant ( $p>0.05$ ).

The immunohistochemical analysis of the neovascularization was performed using an anti-rat antibody against CD34 and the positive endothelial cells were marked brown.
Tab. 3. The microvessel density (MVD) and vascular area in the experimental group

\begin{tabular}{cc}
\hline MVD & Vascular Area (\%) \\
\hline $19.66($ S 4 + C 55) & 0.02 \\
24.33 (S 9 + C 64) & 1.28 \\
20 (S 48 + C 12) & 3.53 \\
17.66 ( S 28 + C 25) & 1.4 \\
11.33 ( S 19 + C 15) & 0.36 \\
\hline
\end{tabular}

$\mathrm{S}$ - stroma; C - capsule

As can be seen in both figures the MVD has not been reduced in the experimental group. MVD between the control group and the experimental group were not statistical significant $(\mathrm{p}>0.05)$ $(32.55 \pm 18.83 ; 18.6 \pm 4.734)$.

In his first study Weidner et al. (1991) used an antibody against factor VIII-related antigen, staining mainly mature vessels and cross-reacting with lymphatic endothelium. After that, recent studies used antibodies directed against platelet endothelial cell adhesion molecule: CD31 or CD34. Both antibodies have the same characteristics with the difference that CD31 can react with fibrobasts and plasma cells. With that consideration we have chosen the CD34 antibody.

Olga Potapova et al. (2006) studied the contribution of individual targets to the antitumor 
efficacy of the sunitinib malate and reached the conclusion that in vivo preclinical mechanism of action of the class III/V RTK inhibitor SU11248 is a combination of direct antitumor cell effects and antiangiogenic inhibition of tumor neovasculature.

In the study performed by Mousseau et al. (2012) they stated that ,orally administered, the combination of sunitinib malate and fingolimod greatly decreased rat breast tumor growth in a syngeneic cancer model (Walker 256). They stated also that these drugs may provide an effective means of reducing tumor angiogenesis, and may improve the delivery of other chemotherapies. In this study sunitinib malate did not have the desired effect by reducing the tumor volumes and reducing the MVD.

\section{CONCLUSIONS}

As a conclusion, the rats treated with Sunitinib malate did not show a lower microvessel density comparative to the untreated ones. The number of rats used in this research was too small and further studies are needed.
Acknowledgments: This paper was published under the frame of European Social Fund, Human Resources Development Operational Programme 2007-2013, projectno. POSDRU/159/1.5/S/136893.

\section{REFERENCES}

1. Adair Thomas H. and Montani Jean-Pierre (2010). Angiogenesis. an Rafael (CA): Morgan \& Claypool Life Sciences

2. Bernard Uzzan, Patrick Nicolas, Michel Cucherat, et al. (2004). Microvessel Density as a Prognostic Factor in Women with Breast Cancer: A Systematic Review of the Literature and Meta-Analysis. Cancer Res 64:2941-2955.

3. Mousseau Yoanne, Séverine Mollard, Karine FaucherDurand, Laurence Richard, Angélique Nizou,Jeanne CookMoreau, Yasser Baaj, Hao Qiu, Xavier Plainard, Laurent Fourcade, Benoit Funalot,Franck G. Sturtz (2012). Fingolimod potentiates the effects of sunitinib malate in a rat breast cancer model Breast Cancer Research and Treatment. Volume 134, Issue 1, pp 31-40

4. Olga Potapova, A. Douglas Laird, Michelle A. Nannini, et al. (2006). Contribution of individual targets to the antitumor efficacy of the multitargeted receptor tyrosine kinase inhibitor SU11248. Mol Cancer Ther 5:1280-1289

5. Weidner N, Semple JP, Welch WR, Folkman J (1991). Tumor angiogenesis and metastasis. Correlation in invasive breast carcinoma. N Engl J Med 324:1- 8. 